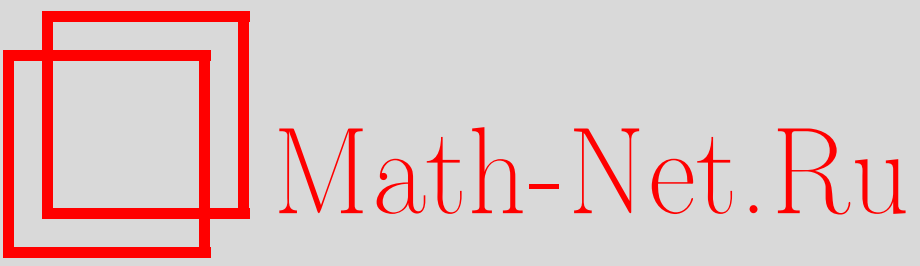

Б. М. Гуревич, А. А. Темпельман, Хаусдорфова размерность и термодинамический формализм, УМH, 1999, том 54, выпуск 2, 171-172

DOI: https://doi.org/10.4213/rm139

Использование Общероссийского математического портала Math-Net.Ru подразумевает, что вы прочитали и согласны с пользовательским соглашением

http://www.mathnet.ru/rus/agreement

Параметры загрузки:

IP : 3.81 .55 .215

26 апреля 2023 г., 15:20:17 


\title{
ХАУСДОРФОВА РАЗМЕРНОСТЬ И ТЕРМОДИНАМИЧЕСКИЙ ФОРМАЛИЗМ
}

\author{
Б. М. ГУРЕВИЧ, А. А. ТЕМПЕЛЬМАН
}

На пространстве $S^{T}=\{x: T \rightarrow S\}$, где $T$ - счетное, а $S$ - конечное множество, можно многими способами ввести метрику, согласованную с топологией, порождаемой цилиндрическими множествами. Мы опишем класс метрик, относительно которых хаусдорфову размерность всего пространства и его частей можно связать с основными понятиями термодинамического формализма [1]. Для ряда частных случаев близкие задачи уже неоднократно рассматривались (см. [2] и указанную там литературу).

1. При любом $V \subset T$ будем называть элементы $x$ множества $S^{V}$ конфигурациями на $V$. Если $V \subset W$, то для каждой конфигурации $x \in S^{W}$ определено ее ограничение $x_{V}$ на $V$. Пусть заданы: последовательность конечных множеств $T_{n} \uparrow T$, непустое $\tau_{c}$-замкнутое множество $X \subset S^{T}$, определенное локально-конечной системой ограничений (ср. $\left.[1 ; \S 1.1]\right)$, и последовательность функций $a_{n}: X \rightarrow \mathbb{R}$, удовлетворяющая условиям: (а) $a_{n}(x)>0$ при всех $x \in X, n \geqslant 1$; (b) $a_{n}(x)=a_{n}\left(x^{\prime}\right)$, если $x_{T_{n}}=x_{T_{n}}^{\prime}$; (с) $a_{n}(x) \downarrow 0$ при $n \rightarrow \infty$ и всех $x \in X$.

Пусть $X_{V}=\left\{x_{V}, x \in X\right\}, V \subset T$, и $C_{n}(x)=\left\{x^{\prime} \in X: x_{T_{n}}=x_{T_{n}}^{\prime}\right\}, x \in X, n \geqslant 1$. Для любых $r>0, b \geqslant r, x \in X$ положим $l(x, r, b)=\max \left\{n \in \mathbb{Z}^{+}: b a_{n}(x) \geqslant r\right\}$. Свойства функций $a_{n}$ гарантируют, что $l(x, r, b)$ корректно определено, причем $\sup _{x} l(x, r, b)<\infty$ при всех $r, b$ и $\inf _{x} l(x, r, b) \rightarrow \infty$ при $r \rightarrow 0$ и всех $b>0$.

Пусть на $X$ задана метрика $\rho$ и $B_{\rho}(x, r)=\left\{x^{\prime} \in X: \rho\left(x, x^{\prime}\right)<r\right\}, r>0, x \in X$. При $n \in \mathbb{N}, b>0$ поставим в соответствие каждой точке $y$ шара $B \rho\left(x, b a_{n}(x)\right)$ цилиндр $C_{n(y)}(y)$, где $n(y)=l\left(y, b a_{n}(x), b\right)$. Пусть $L_{\rho}(x, n, b)$ - максимальное из таких $l$, что среди этих цилиндров существует $l$ попарно не пересекающихся. Определим индекс Морана равенством $L_{\rho}\left(\left\{a_{n}\right\}, b\right)=\sup _{x, n} L_{\rho}(x, n, b)$ (cp. [2], [3]). Назовем метрику $\rho$ согласованной $c$ последовательностью $\left\{a_{n}\right\}$, если существуют такие $b_{1}, b_{2}>0$, что (i) $\rho\left(x, x^{\prime}\right) \leqslant b_{1} a_{n}(x)$ при $x_{T_{n}}=x_{T_{n}}^{\prime}$ и (ii) $L_{\rho}\left(\left\{a_{n}\right\}, b_{2}\right)<\infty$.

Естественный класс таких метрик получается с помошью геометрических конструкций, отвечающих $X$ и $\left\{a_{n}\right\}$ (см. [2], [3]).

Введем теперь класс последовательностей $\left\{a_{n}\right\}$, связанных с моделями статистической физики на $T$. Эти модели задаются потенциалами (см. [1], [4]). Пусть $\mathscr{F}$ - совокупность конечных множеств $F \subset T$ и $X^{0}=\bigcup_{V \in \mathscr{F}} X_{V}$. Будем считать потенциал $U$ функцией, заданной на $X^{0}$, и писать $U(V, x)$ вместо $U(x), x \in X_{V}, V \in \mathscr{F}$. Предположив, что $\|U\|<\infty$, где

$$
\|U\|=\sup _{t \in T} \sum_{V: t \in V \in \mathscr{F}} \max _{x \in X_{V}}|U(V, x)|,
$$

обозначим через $E^{(U)}(V, x), Z^{(U)}(V)$ и $E^{(U)}\left(V, x ; \bar{V}, x_{\bar{V}}\right)$ соответственно әнергию конфигураuии $x \in X_{V}, V \in \mathscr{F}$, статистическую сумму в "обвеме" $V$ (с пустыми граничными условиями) и әнергию взаимодействия конфигурации $x_{V}$ и конфигурации $x_{\bar{V}}$, где $x \in X$ и $\bar{V}=T \backslash V$. Предел $\lim _{n \rightarrow \infty}\left(\operatorname{card}\left(T_{n}\right)\right)^{-1} \ln Z^{(U)}\left(T_{n}\right)=P\left(U,\left\{T_{n}\right\}\right)$, если он существует, будем назьвать давлением, отвечающим потенциалу $U$ и последовательности множеств $\left\{T_{n}\right\}$.

Нам понадобятся следуюшие условия (последнее из которых было с иной целью сформулировано Рюэлем [1]):

(A) $\varliminf_{n \rightarrow \infty}\left(\operatorname{card}\left(T_{n}\right)\right)^{-1} \inf _{x \in X} E^{(U)}\left(T_{n}, x_{T_{n}}\right)>0$;

(B) $\lim _{n \rightarrow \infty}\left(\operatorname{card}\left(T_{n}\right)\right)^{-1} \sup _{x \in X}\left|E^{(U)}\left(T_{n}, x_{T_{n}} ; \bar{T}_{n}, x_{\bar{T}_{n}}\right)\right|=0$;

(C) $\lim _{n \rightarrow \infty} \operatorname{card}\left(T_{n+1}\right) / \operatorname{card}\left(T_{n}\right)=1$;

(D) существует такая последовательность множеств $T_{n}^{\prime} \subset T, n=1,2, \ldots$, что $T_{n} \subset T_{n}^{\prime}, \lim _{n \rightarrow \infty} \operatorname{card}\left(T_{n}^{\prime}\right) / \operatorname{card}\left(T_{n}\right)=1$ и для любых $x, y \in X$ найдется $z \in X c$ $z_{T_{n}}=x_{T_{n}}, \overrightarrow{z_{T}} \underset{T T_{n}^{\prime}}{ }=y_{T_{n} \backslash T_{n}^{\prime}}$. 
Легко видеть, что при выполнении условия $($ А) последовательность функций

$$
a_{n}^{(U)}(x)=\exp \left[-E^{(U)}\left(T_{n}, x_{T_{n}}\right)\right], \quad n \in \mathbb{N}, \quad x \in X,
$$

удовлетворяет условиям (a)-(c).

Теорема 1. Если $\|U\|<\infty$, выполняется условие (А) и найдется $\beta_{0} \geqslant 0$, для которого давление $P\left(\beta_{0} U,\left\{T_{n}\right\}\right)$ существует и равно нулю, то для любой метрики $\rho$, согласованной с последовательностью функций $a_{n}^{(U)}$, хаусдорфова размерность $d_{H}(X, \rho)$ множества $X$ не превосходит $\beta_{0}$; если, кроме того, выполняются условия (B)-(D), $\bmod _{H}(X, \rho)=\beta_{0}$.

ЗАмечАнИЕ 1. Можно показать, что при условиях (A)-(D) хаусдорфова размерность всякой гиббсовской меры с потенциалом $U$ равна $d_{H}(X, \rho)$.

2. Рассмотрим более конкретную ситуацию. Пусть $T=\mathbb{Z}^{d}, d \geqslant 1$. Для любых $V \subset T$, $t \in T$ определим отображение $\sigma_{V}^{t}: S^{V} \rightarrow S^{V-t}$ равенством $\left(\sigma_{V}^{t} x\right)\left(t^{\prime}\right)=x\left(t+t^{\prime}\right), t^{\prime} \in V-t$, $x \in S^{V}$. Положим $\sigma=\left\{\sigma_{T}^{t}, t \in T\right\}$. Пусть множество $X$ из п. 1 непусто и $\sigma$-инвариантно, а потенциал $U: X^{0} \rightarrow \mathbb{R}$ инвариантен в том смысле, что $U(V+t, x)=U\left(V, \sigma_{V+t}^{t} x\right), t \in T$, $V \in \mathscr{F}, x \in X_{V+t}$.

ПреДЛоЖениЕ. Если $U(V, x) \geqslant 0$ nри всеx $V \in \mathscr{F}, x \in X_{V}$ u найдется такое $V^{+} \in \mathscr{F}$, что $E^{(U)}\left(V^{+}, x\right)>0$ при всех $x \in X_{V^{+}}$(локальная положительность $U$ ), то выполняется условие (A).

Назовем последовательность $T_{n} \uparrow T$ регулярной, если $\lim _{n \rightarrow \infty} \operatorname{card}\left(T_{n} \Delta\left(T_{n}+t\right)\right) / \operatorname{card}\left(T_{n}\right)$ $=0$ при всех $t \in T$ и $\sup _{n} \operatorname{card}\left(t-t^{\prime}, t, t^{\prime} \in T_{n}\right) / \operatorname{card}\left(T_{n}\right)<\infty$.

Теорема 2. Пусть регулярная последовательность $\left\{T_{n}\right\}$ удовлетворяет условию (C), а потенциал $U$ c $\|U\|<\infty$ инвариантен и локально положителен. Тогда для любой метрики $\rho$ на $X$, согласованной с последовательностью $\left\{a_{n}^{(U)}\right\}$, вьполняется соотношение Боуэна $P\left(d_{H}(X, \rho) U\right)=0$.

\section{СПИСОК ЛИТЕРАТУРЫ}

[1] Ruelle D. Thermodynamic Formalism. Reading, Massachusetts: Addison-Wesley, 1978. [2] Pesin Ya. B. Dimension Theory in Dynamical Systems. Chicago: Chicago Univ. Press, 1997. [3] Pesin Ya. B., Weiss H. // Commun. Math. Phys. 1996. V. 182. P. 105-153. [4] Гeopги Х.-О. Гиббсовские меры и фазовые переходы. М.: Мир, 1992.

Московский государственный

Принято редколлегией университет им. М. В. Ломоносова; 26.01.1999

Университет штата Пенсильвания, США

E-mail: bmg@gibbs.math.msu.su arkady@stat.psu.edu 